Original Article - Clinical Science

\title{
Distribution and progression of add power among people in need of near correction
}

\author{
Xiaotong Han MD, ${ }^{1,2}$ Pei Ying Lee BOptom, ${ }^{2}$ Chi Liu MSc ${ }^{3}$ and Mingguang He MD \\ $\mathrm{PhD}^{1,2}$
}

1. State Key Laboratory of Ophthalmology, Zhongshan Ophthalmic Center, Sun Yat-sen University, Guangzhou, China.

2. Centre for Eye Research Australia; Ophthalmology, Department of Surgery, University of Melbourne, Melbourne, Australia.

3. Guangzhou Healgoo Interactive Medical Technology Co. Ltd., Guangzhou, China.

Correspondence: Mingguang He, MD, PhD, Zhongshan Ophthalmic Center, Guangzhou, People's Republic of China. Email: mingquang he@yahoo.com

Short running title: Distribution and progression of add power

Received 11 January 2018; accepted 15 March 2018

Conflict of interest: None

This is the author manuscript accepted for publication and has undergone full peer review but has not been through the copyediting, typesetting, pagination and proofreading process, which may lead to differences between this version and the Version of Record. Please cite this article as doi: 10.1111/ceo.13301

This article is protected by copyright. All rights reserved. 
Funding sources: This study was supported by the World Health Organization, Geneva, Switzerland (under National Institutes of Health, Bethesda, MD, contract No. N01-EY-2103) and the by Fundamental Research Funds of the State Key Laboratory of Ophthalmology at the Zhongshan Ophthalmic Center. Prof. He receives support from the University of Melbourne at Research Accelerator Program and the CERA Foundation. The Centre for Eye Research Australia receives Operational Infrastructure Support from the Victorian State Government. The sponsor or funding organization had no role in the design or conduct of this research. 


\section{ABSTRACT}

Importance: This study helps to better understand the need and trend in presbyopic add power in the aging society.

Background: Distribution and progression of presbyopic add power in East Asian population is largely unknown.

Design: Prospective cohort study.

Participants: 303 participants from a population-based study of residents aged 35 years and older in Guangzhou, China.

Methods: Visual acuity (VA) test and non-cycloplegic automated refraction were performed at baseline in 2008 and the 6-year follow-up per standardized protocol. Participants with presenting near VA $\leq 20 / 40$ underwent distance subjective refraction and add power measurement by increasing plus lens at a standard distance of $40 \mathrm{~cm}$ at each visit.

Main outcome measures: add power at baseline and follow-ups.

Results: Mean (standard deviation) age of the study participants was 57.6 (11.1) years and $50.2 \%$ were female. The mean add power at baseline was $1.43,1.73,2.03$ and 2.20 diopters (D) for individuals in the age groups of $35-44,45-54,55-64$ and $65+$ years, respectively. Participants with older age and lower educational level had significantly higher add power requirements $(P<0.001)$. The overall 6-year increase in add power was $0.15 \mathrm{D}$ ( $95 \% \mathrm{CI}: 0.06$ to 0.25 ), and was smaller in myopic subjects 
$(P=0.03)$. Baseline age and add power, but not changes in biometric factors, were associated with longitudinal change in add power $(P<0.001)$.

Conclusions and relevance: Distribution and progression of add power in Chinese was different from that previously suggested by Caucasian studies. More studies are needed to establish up-to-date age-related add power prescription norms for population of different ethnicities.

Keywords: add power, presbyopia, longitudinal, epidemiology 


\section{INTRODUCTION}

Presbyopia is a progressing and inevitable condition with age. It is estimated that 1.27 billion people worldwide had presbyopia in 2010, with a potential productivity loss of US $\$ 11.02$ billion. ${ }^{1}$ Reported prevalence of presbyopia for people aged 40 years or older ranges from $43.8 \%$ in Asian countries to more than $80 \%$ in Western countries, however little is known about the progression of presbyopia with age. ${ }^{23}$

It is a common practice for ophthalmologists and optometrists to prescribe add power, which is a spherical positive power superimposed on fully-corrected distance refraction, for patients with presbyopia to aid near vision. Thus changes in add power can partly reflect the progression of presbyopia from a clinical perspective. Donders, Duane, Hofstetter were the first to describe the age-related norms of accommodation amplitude

while Pointer provided the add powers for different age groups, which are still used as reference in clinics today. ${ }^{4-6}$ However, these norms were mostly based on Caucasian populations and its applicability to other populations were largely unknown.

Furthermore, whether Hofstetter and Pointer's norms are still valid nowadays is unknown. Sociodemographic and environmental factors such as refractive error and 
temperature had been reported to associate with presbyopia onset. ${ }^{78}$ Therefore changes in the last decades, for example the increasing prevalence of myopia in East Asia, might lead to significant cohort effect.

To the best of our knowledge, the age-related add power requirement for East Asian population as well as the longitudinal progression of add power have never been reported. Thus purposes of this study were to investigate: (1) the age-related requirement of add power in a Chinese population; (2) the 6-year longitudinal change in add power; and (3) risk factors for longitudinal change in add power.

\section{METHODS}

\section{Study Population}

Details of the recruitment and study methodology of the population-based study conducted in Guangzhou, China had been reported elsewhere. ${ }^{9}$ Briefly, 1817 subjects aged 35 years or older participated in the baseline study in 2008 and all were invited for the 2-year (2010) and 6-year (2014) follow-up examinations. A subset of participants from this cohort with available add power measurements at both baseline and the 2014 follow-up examinations were included in the current study. Participants who had undergone refractive surgeries or were aphakic or pseudophakic at baseline or during the follow-up examination were excluded. Eyes with presenting distance 
visual acuity (VA) $\leq 20 / 200$ at either examination were also excluded. The study protocol was approved by the World Health Organization Secretariat Committee on Research Involving Human Subjects and by the institutional review board at Zhongshan Ophthalmic Center (ZOC) in Guangzhou, China. Approval for the follow-up survey was also obtained from the ZOC review ethics committee. Written informed consent was obtained from all participants and the study was conducted in accordance with the tenets of the Declaration of Helsinki. The participants did not receive any financial compensation.

\section{Procedures}

Questionnaires including information on age, gender, education background and detailed ophthalmic surgical history were administered by trained nurses at both baseline and follow-up examinations. Distance and near VA were measured indoor under ambient lighting with a LogMAR ETDRS tumbling E chart (Precision Vision, La Salle, Illinois, USA) per standardized protocol. Non-cycloplegic automated refraction was carried out for all participants at baseline and follow-up examinations using an auto kerato-refractometer (KR-8800; Topcon Corp, Tokyo, Japan). Five consecutive measurements were performed for each eye and the mean was recorded as the final value. Corneal refractive power was also measured and the mean of five readings was recorded for each eye. Participants with presenting near vision impairment (NVI), defined as presenting near VA of $\leq 20 / 40$, were further examined for add power at a 
standard testing distance of $40 \mathrm{~cm}$ after fully corrected for distance refractive error based on subjective refraction at both baseline and follow-up examinations. Plus lens was added at an increment of $+0.25 \mathrm{D}$ and the minimum add power needed to obtain best-corrected near VA was recorded for each eye. Binocular add power was determined by the smaller add power in either eye.

Anterior chamber depth (ACD) was measured in each eye using a non-contact partial coherence laser interferometry (IOLMaster, version 3.0, Carl Zeiss Meditec at baseline and Lenstar LS900, Haag-Streit AG, Switzerland at the 2014 follow-up) in a dark room (illumination $<5$ lux). Measurements with a ratio of signal to noise of less than 2.0 or those that differed from other values by more than $0.1 \mathrm{~mm}$ were re-measured. The mean of 5 measurements was recorded as the final result. In the 2010 follow up study, both IOLMaster and Lenstar LS900 were used to measure ACD. We found that the ACD data measured by IOLMaster correlated well with the measurement obtained using Lenstar, therefore an equation is generated to relate these values. This allowed us to convert the ACD data measured by Lenstar in 2014 into equivalent values for comparison with the baseline ACD data measured by IOLMaster.

Lens thickness (LT) was measured by anterior segment optical coherence tomography (AS-OCT) imaging (Visante; Carl Zeiss Meditec) at baseline and by non-contact partial coherence laser interferometry (Lenstar LS900, Haag-Streit AG, Switzerland) in 2014. 
Similar to the ACD measurements, both equipment were used in the 2010 follow up study to measure LT and the data correlated well. An equation was generated to convert the LT data from 2014 for comparison with the baseline LT. Change in ACD, LT and corneal power were defined as the corresponding values at baseline subtracted from the values at the 2014 follow-up.

\section{Data Management and Analyses}

Spherical equivalent (SE), calculated as spherical power plus half of cylindrical power, was used to represent refraction. Baseline refractive state was categorized into myopia (SE < -0.5 diopter, D), emmetropia (-0.5D $\leq$ SE $\leq+0.5 \mathrm{D}$ ) and hyperopia (SE > $+0.5 \mathrm{D})$. Age was categorized into four age groups: $35-44,45-54,55-64$ and $\geq 65$, based on the age obtained at baseline. Education level was dichotomized as less than high school and high school or above.

All statistical analyses were performed using STATA Statistical Software: Release 12.0 (StataCorp LP, Colleage Station, TX). Group t-test and trend-analysis were used to assess the difference in baseline add power and the 6-year longitudinal changes in add power between different age groups, gender, education background and baseline SE status. Univariate and stepwise multiple regression (entry $\mathrm{P}$ value $=0.05$ ) were used to analyse the association between longitudinal changes in add power and potential risk factors. P-values of $\leq 0.05$ were considered statistically significant. 


\section{RESULTS}

A total of 346 participants underwent add power measurement at both baseline and the 2014 follow-up examination. Two participants with poor presenting distance VA and 41 participants who had undergone refractive surgery or with aphakic or pseudophakic eyes were excluded. Thus, a total of 303 participants were included in the final analysis. The mean (standard deviation, SD) age was 57.6 (11.1) years and $50.2 \%$ were female.

Table 1 shows the add power required at baseline for different age, gender, education and refractive state groups. The mean (SD) required add power in this population ranged from $1.43(0.91) \mathrm{D}$ in the 35 to 44 age group to $2.20(0.85) \mathrm{D}$ in the $\geq 65$ age group. There was a significant increase in add power with age $(P<0.001)$. Participants with lower educational level were also found to require higher add power $(P<0.001)$. There was no significant association between gender or baseline refractive state and add power. In addition, there was a rapid increase in add power requirement from age 40 to 50 years and slowed down afterwards, as shown in Figure 1.

Table 1: Add power requirement at baseline 


\begin{tabular}{lccc}
\hline Characteristics & N & $\begin{array}{c}\text { Add power } \\
\text { (mean } \pm \text { SD), D }\end{array}$ & P-value \\
\hline Age at Baseline & 24 & $1.43 \pm 0.91$ & $<0.001$ \\
$35-44$ & 125 & $1.73 \pm 0.55$ & \\
$45-54$ & 71 & $2.03 \pm 0.66$ & \\
$55-64$ & 83 & $2.20 \pm 0.85$ & \\
$\geq 65$ & & & \\
Sex & 151 & $1.84 \pm 0.69$ & 0.12 \\
$\quad$ Male & 152 & $1.97 \pm 0.78$ & \\
Female & & & \\
Education & 157 & $2.05 \pm 0.76$ & $<0.001$ \\
$\quad$ Less than high school & 146 & $1.76 \pm 0.69$ & \\
High school or above & & & 0.07 \\
Baseline refractive state & & $2.01 \pm 0.87$ & \\
Mild myopia & 80 & $1.89 \pm 0.70$ & \\
Emmetropia & 110 & $1.85 \pm 0.68$ & \\
Hyperopia & 303 & $1.91 \pm 0.74$ & \\
ALL & & & \\
\hline
\end{tabular}

D: diopter; SD: standard deviation. 


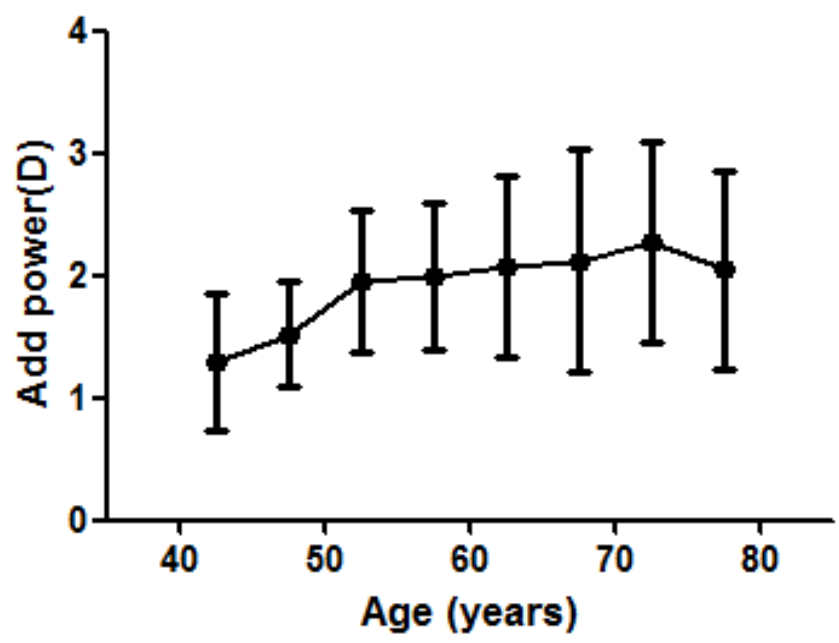

Figure 1: Add power requirements in different age groups at baseline.

The 6-year changes in add power by baseline age, gender, education and refractive state is detailed in Table 2 . The overall increase in add power was $0.15 \mathrm{D}(95 \%$ confidence interval $[\mathrm{CI}]: 0.06$ to 0.25 ) and was more significant in participants with more hyperopic refraction $(\mathrm{P}=0.03)$. Age, gender and education background were not associated with the longitudinal change in add power.

Table 2: Six-year changes of add power

\begin{tabular}{lcc}
\hline Characteristics & N $\begin{array}{c}\text { Change in add power } \\
\text { mean }(95 \% \mathrm{CI}), \mathrm{D}\end{array}$ & P-value \\
\hline
\end{tabular}

Age at Baseline

$35-44$

$24 \quad 0.08(-0.44$ to 0.61$)$

0.77 


$\begin{array}{lcc}45-54 & 125 & 0.15(0.05 \text { to } 0.25) \\ 55-64 & 71 & 0.21(0.05 \text { to } 0.37) \\ \geq 65 & 83 & 0.13(-0.12 \text { to } 0.38)\end{array}$

Sex

Male

$151 \quad 0.17(0.03$ to 0.30$)$

0.81

Female

$152 \quad 0.14(0.01$ to 0.28$)$

\section{Education}

Less than high school

$157 \quad 0.15(0.01$ to 0.28$)$

0.89

High school or above

146

$0.16(0.03$ to 0.30$)$

\section{Baseline refractive state}

Mild myopia

Emmetropia

Hyperopia
80

110

113
$0.05(-0.19$ to 0.29$) \quad 0.03$

$0.12(-0.02$ to 0.26$)$

$0.26(0.12$ to 0.40$)$

$303 \quad 0.15(0.06$ to 0.25$)$

D: diopter; CI: confidence interval.

Table 3 shows the association between potential risk factors and 6-year change in add power. Older age and lower add power requirements at baseline were both associated with an increase in longitudinal add power change $(P<0.001)$, which in total could explain $72.8 \%$ of the variance. Baseline refractive error was associated with longitudinal add power change in the univariate analysis $(P=0.03)$ but not in the multiple regression analysis. Biometric factors, including change in corneal power, ACD and LT were not associated with longitudinal add power changes.

Table 3: Association of potential risk factors with 6-year changes in add power by linear 
regression

\begin{tabular}{|c|c|c|c|c|}
\hline \multirow[b]{2}{*}{ Factors } & \multicolumn{2}{|c|}{ Univariate regression } & \multicolumn{2}{|c|}{$\begin{array}{c}\text { Multiple regression } \\
\text { model }\end{array}$} \\
\hline & $\begin{array}{c}\text { Regression } \\
\text { coefficient } \\
\text { Mean }(95 \% \mathrm{CI})\end{array}$ & $\begin{array}{c}\text { P } \\
\text { Value }\end{array}$ & $\begin{array}{c}\text { Regression } \\
\text { coefficient } \\
\text { Mean }(95 \% \mathrm{CI})\end{array}$ & $\begin{array}{c}\mathbf{P} \\
\text { Value }\end{array}$ \\
\hline Age, years & $\begin{array}{c}-0.002(-0.01 \text { to } \\
0.01)\end{array}$ & 0.58 & $0.02(0.02$ to 0.03$)$ & $<0.001$ \\
\hline Sex, female & $\begin{array}{c}-0.02(-0.21 \text { to } \\
0.17)\end{array}$ & 0.81 & - & - \\
\hline $\begin{array}{l}\text { Education level, high } \\
\text { school or above }\end{array}$ & $0.01(-0.18$ to 0.20$)$ & 0.89 & - & - \\
\hline Baseline SE, D & $\begin{array}{c}0.04(0.004 \text { to } \\
0.07)\end{array}$ & 0.03 & - & - \\
\hline Baseline add power, D & $\begin{array}{c}-0.87(-0.96 \text { to } \\
-0.79)\end{array}$ & $<0.001$ & $\begin{array}{c}-0.95(-1.05 \text { to } \\
-0.85)\end{array}$ & $<0.001$ \\
\hline$\triangle \mathrm{ACD}, \mathrm{mm}$ & $\begin{array}{c}-0.14(-1.00 \text { to } \\
0.72)\end{array}$ & 0.75 & - & - \\
\hline$\Delta$ Corneal power, $\mathrm{D}$ & $\begin{array}{c}-0.07(-0.27 \text { to } \\
0.13)\end{array}$ & 0.49 & - & - \\
\hline$\Delta \mathrm{LT}, \mathrm{mm}$ & $0.13(-0.44$ to 0.69$)$ & 0.66 & - & - \\
\hline
\end{tabular}

SE: spherical equivalence; ACD: anterior chamber depth; LT: lens thickness; D: diopter; CI: confidence interval.

Adjusted $\mathrm{R}^{2}$ for multiple regression model $=72.8 \%$

\section{DISCUSSION}


The current study presents the age-related add power requirements in a Chinese population with presenting NVI, as well as the 6-year longitudinal changes and risk factors of add power. Subjects with presenting NVI are the cohort of the population who are more likely to attend an eye examination for near vision correction compared to those without NVI, thus we believe our result could offer pragmatic information to clinical practice. The authors are unaware of any other report on age-related add power norms in East Asian population or longitudinal add power changes.

Our study found an earlier onset of presbyopia compared to the norms recommended by Hofstetter and Pointer (Supplement Fig1). The fact that their formulas were mostly based on Caucasian populations had led to doubt by subsequent studies which identified significant associations between ethnicity and presbyopia. Africans were found to require reading add power 5 to 10 years earlier than Caucasians. ${ }^{10}$ Both Hispanic and non-Hispanic population from Los Angeles had a later onset and slower progression of presbyopia than the Hofstetter's norms. ${ }^{11}$ The Shahroud Eye Cohort Study showed the required add power was $0.5 \mathrm{D}$ less than the Pointer's recommendation in an Iranian population. ${ }^{12}$ A study of 121 Hong Kong Chinese subjects showed that Chinese have lower amplitudes of accommodation and thus need higher add powers compared to Caucasians. ${ }^{13}$ Besides that, social and environmental factors including temperature, occupation and nutrition can influence the development of presbyopia, which could lead to a drastic change in the norm of 
age-related add power requirements in the recent decades. For example, people living in warmer areas were reported to have an earlier onset of presbyopia and farmers had a later onset of presbyopia than housewives or construction workers. ${ }^{814}$ Thus more up-to-date norms of age-related add power are needed for populations of different ethnicities.

Most cross-sectional studies of Caucasian and African populations reported that women had an earlier onset for presbyopia. It was suggested to be due to difference in task forms and viewing distances, as well as menopause. ${ }^{15-17}$ However, two studies based on Asian populations found no gender difference or a higher risk of presbyopia in men. ${ }^{218}$ Gender difference in add power requirements were only reported by Hofstetter in 1949 and Pointer in 1995, and women were found to have a higher need of add powers compared to men of the same age.${ }^{15}$ However in our study, there was no gender difference in add power requirements at baseline, and the longitudinal change in add power was also similar between genders. This further supports the existence of ethnic difference and more studies are needed to illustrate whether gender difference for add power exists in East Asian populations. Our study also found that participants with lower educational level had significantly higher add power requirements, this could be simply due to a higher proportion of older subjects in this subgroup (proportion of subjects aged 55 years or above: <high school, $66.2 \%$; $\geq$ high school, $33.2 \%)$. 
To the best of our knowledge, there was no longitudinal study addressing cohort effect on add power. Previously-reported progression rate of add power based on cross-sectional data was roughly $0.1 \mathrm{D} / \mathrm{year}$, which is consistent with our cross-sectional results. ${ }^{612}$ However our longitudinal analysis showed an average change of $0.025 \mathrm{D} /$ year and even lower in the youngest cohorts (35-44 years), which might be explained by higher prevalence of myopia in this cohort $(45.8 \%$ among $35-44$ years group versus $24.7 \%$ in $\geq 45$ years group) while table 2 documents a lower add power progression among myopes (Table 2). ${ }^{19}$

The most important mechanism underlying presbyopia progression was believed to be the increase in lenticular stiffness with accompanying reduced elasticity based on the Helmholtz theory. It was suggested that ciliary muscles, zonules and vitreous also played a role in presbyopia mechanism but this was proven to have very limited effect based on mathematical or animal models. ${ }^{20-22}$ Our analysis showed that longitudinal change in add power was related to baseline age and add power, but not changes in anterior chamber biometric factors. More myopic refractive error was related to less add power progression in univariate model but not in the multiple regression model, which could be due to lack of statistical power or interaction effect between baseline refractive error and add power. This could further enhance our understanding of presbyopia progression from an epidemiological point of view. 
Limitations of the current study included a relatively small sample and only subjects with presenting NVI were included, but our result could be of practical clinical significance as presbyopic patients without presenting NVI are less likely to present to eye clinics. Lack of data on accommodation amplitude could be another potential limitation, however add power is a more comprehensive indicator, and the distribution and progression of add power can better reflect the true eye care need.

In conclusion, our study presented for the first time the norm of age-related add power and its longitudinal changes in a Chinese population with presenting NVI. There was no gender difference, but a significant cohort effect in add power requirements. More studies are needed to establish up-to-date recommendations of age-related add power prescription for different populations.

\section{REFERENCES}

1. Frick KD, Joy SM, Wilson DA, et al. The Global Burden of Potential Productivity Loss from Uncorrected Presbyopia. Ophthalmology 2015;122(8):1706-10. doi: 10.1016/j.ophtha.2015.04.014 
2. Kidd Man RE, Fenwick EK, Sabanayagam C, et al. Prevalence, Correlates, and Impact of Uncorrected Presbyopia in a Multiethnic Asian Population. American journal of ophthalmology 2016;168:191-200. doi: 10.1016/j.ajo.2016.05.019

3. Burke AG, Patel I, Munoz B, et al. Population-based study of presbyopia in rural Tanzania. Ophthalmology 2006;113(5):723-7. doi:

10.1016/j.ophtha.2006.01.030

4. Duane A. Normal values of the accommodation at all ages. J Amer Med Assoc 1912;59:1010-13.

5. HW. H. A useful table for age and amplitude. Optom World 1950;38:42-5.

6. Pointer JS. The Presbyopic Add .1. Magnitude And Distribution In a Historical Context. Ophthal Physl Opt 1995;15(4):235-40. doi: Doi $10.1016 / 0275-5408(94) 00021-Q$

7. Nirmalan PK, Krishnaiah S, Shamanna BR, et al. A population-based assessment of presbyopia in the state of Andhra Pradesh, south India: the Andhra Pradesh Eye Disease Study. Investigative ophthalmology \& visual science 2006;47(6):2324-8. doi: 10.1167/iovs.05-1192

8. Miranda MN. The geographic factor in the onset of presbyopia. Transactions of the American Ophthalmological Society 1979;77:603-21.

9. Han X, Ellwein LB, Guo X, et al. Progression of Near Vision Loss and Incidence of Near Vision Impairment in an Adult Chinese Population. Ophthalmology 2017 doi: $10.1016 /$ j.ophtha.2017.01.020 
10. Covell LL. Presbyopia; comparative observations of white and Negro populations. American journal of ophthalmology 1950;33(8):1275-6.

11. Carnevali T, Southaphanh P. A retrospective study on presbyopia onset and progression in a Hispanic population. Optometry 2005;76(1):37-46.

12. Hashemi H, Khabazkhoob M, Jafarzadehpur $\mathrm{E}$, et al. Population-based study of presbyopia in Shahroud, Iran. Clin Exp Ophthalmo/2012;40(9):863-8. doi: 10.1111/j.1442-9071.2012.02799.x

13. Edwards MH, Law LF, Lee CM, et al. Clinical norms for amplitude of accommodation in Chinese. Ophthalmic \& physiological optics : the journal of the British College of Ophthalmic Opticians 1993;13(2):199-204.

14. Garcia Serrano JL, Lopez Raya R, Mylonopoulos Caripidis T. [Variables related to the first presbyopia correction]. Archivos de la Sociedad Espanola de Oftalmologia 2002;77(11):597-604.

15. Hickenbotham A, Roorda A, Steinmaus C, et al. Meta-analysis of sex differences in presbyopia. Investigative ophthalmology \& visual science 2012;53(6):3215-20. doi: $10.1167 /$ iovs.12-9791

16. Marmamula S, Narsaiah S, Shekhar K, et al. Presbyopia, spectacles use and spectacle correction coverage for near vision among cloth weaving communities in Prakasam district in South India. Ophthal Physl Opt 2013;33(5):597-603. doi: 10.1111/opo.12079 
17. Pointer JS. The Presbyopic Add .2. Age-Related Trend And a Gender Difference. Ophthal Physl Opt 1995;15(4):241-48. doi: DOI 10.1046/j.1475-1313.1995.9400022r.x

18. Lu Q, He W, Murthy GV, et al. Presbyopia and near-vision impairment in rural northern China. Investigative ophthalmology \& visual science 2011;52(5):2300-5. doi: 10.1167/iovs.10-6569

19. Holden BA, Fricke TR, Wilson DA, et al. Global Prevalence of Myopia and High Myopia and Temporal Trends from 2000 through 2050. Ophthalmology 2016 doi: 10.1016/j.ophtha.2016.01.006

20. Truscott RJ. Presbyopia. Emerging from a blur towards an understanding of the molecular basis for this most common eye condition. Experimental eye research 2009;88(2):241-7. doi: 10.1016/j.exer.2008.07.003

21. Laughton DS, Sheppard AL, Davies LN. A longitudinal study of accommodative changes in biometry during incipient presbyopia. Ophthalmic \& physiological optics : the journal of the British College of Ophthalmic Opticians 2016;36(1):33-42. doi: 10.1111/opo.12242

22. Lopez-Alcon D, Marin-Franch I, Fernandez-Sanchez V, et al. Optical factors influencing the amplitude of accommodation. Vision research 2016 doi: 10.1016/j.visres.2016.09.003

\section{SUPPLEMENTARY FIGURE LEGEND}


Supplementary figure 1: Comparison of age-related add power requirements by Hofstetter, Pointer and our study 
.4 HHH

Fig1.tif

This article is protected by copyright. All rights reserved. 


\section{University Library}

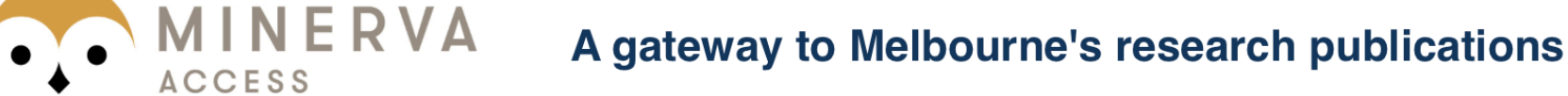

Minerva Access is the Institutional Repository of The University of Melbourne

Author/s:

Han, X;Lee, PY;Liu, C;He, M

Title:

Distribution and progression of add power among people in need of near correction

Date:

2018-11-01

Citation:

Han, X., Lee, P. Y., Liu, C. \& He, M. (2018). Distribution and progression of add power among people in need of near correction. CLINICAL AND EXPERIMENTAL OPHTHALMOLOGY, 46 (8), pp.882-887. https://doi.org/10.1111/ceo.13301.

Persistent Link:

http://hdl.handle.net/11343/283822 